\title{
Toxicidade aguda de inseticidas e espalhante usados no controle de vetores para o bioindicador caramujo (Pomacea canaliculata)
}

\section{Acute toxicity of insecticides and spreader used in vectors control for bioindicator snail (Pomacea canaliculata)}

Karollina Pereira Leonel ${ }^{1}$, Juliana Mattos Morandi ${ }^{1}$, Isabella Alves Brunetti ${ }^{1}$, Lays Drubi Brait Aparicio ${ }^{1}$, Claudinei da Cruz ${ }^{1}$

${ }^{1}$ Laboratório de Ecotoxicologia e Eficácia dos Agrotóxicos (LEEA), Centro Universitário da Fundação Educacional de Barretos - Barretos (SP), Brasil.

\begin{abstract}
Resumo
O controle das doenças vetoriais é um grande desafio de saúde pública e um dos problemas da utilização do controle químico é a poluição ambiental causada por produtos químicos domissanitários. Assim, os objetivos deste estudo foram determinar a toxicidade aguda (CE50;48 h) de deltametrina, lambda-cialotrina (duas formulações), alfacipermetrina e do espalhante adesivo à base de óleo vegetal para o bioindicador de exposição, o caramujo (Pomacea canaliculata). Os ensaios de toxicidade aguda foram realizados de acordo com a adaptação de normas nacionais de ensaio para organismos aquáticos. A toxicidade aguda (CE50;48 h) de lambda-cialotrina foi superior a 118,0 mg.L.-1 (duas formulações), sendo classificada como praticamente não tóxica. Para deltametrina, a CE50;48 h foi de $0,45 \mathrm{mg} . \mathrm{L}^{-1}$ (muito tóxica), para a alfacipermetrina, de $28,76 \mathrm{mg} . \mathrm{L}^{-1}$ e para o óleo vegetal, de $70,62 \mathrm{mg} . \mathrm{L}^{-1}$ (pouco tóxica). O P. canaliculata pode ser utilizado no biomonitoramento de inseticidas e produtos domissanitários usados no controle de vetores, tendo ele apresentado excelente resposta de sensibilidade para deltametrina $>$ alfacipermetrina $>$ óleo vegetal $>$ lambda-cialotrina $=$ lambda-cialotrina (duas formulações). $\mathrm{O}$ caramujo $P$. canaliculata pode ser empregado no biomonitoramento de inseticidas e espalhante adesivo utilizados no controle de vetores, com resposta sensível aos produtos testados.
\end{abstract}

Palavras-chave: biomonitoramento; segurança ambiental; saúde pública; produtos domissanitários.

\begin{abstract}
Control of vector-borne diseases is a major public health issue, and one of the problems of using chemical control is the environmental pollution caused by household cleaning chemicals. The objectives of this study were to determine the acute toxicity (EC50, $48 \mathrm{~h}$ ) of Deltamethrin, Lambda-cyhalothrin (two formulations), Alpha-cypermethrin, and spreader-sticker vegetable oil for the snail (Pomacea canaliculata) as a bioindicator of exposure. Acute toxicity tests were performed by adapting national testing standards for aquatic organisms. Acute toxicity (EC50;48h) for lambda-cyhalothrin was above $118.0 \mathrm{mg} \mathrm{L}^{-1}$ (two formulations), being classified as virtually nontoxic. For EC50;48h deltamethrin, it was of $0.45 \mathrm{mg} \mathrm{L}^{-1}$ (highly toxic); for Alpha-cypermethrin, $28.76 \mathrm{mg} \mathrm{L}^{-1}$; and for the vegetable oil, $70.62 \mathrm{mg} \mathrm{L}^{-1}$ (slightly toxic). P. canaliculata can be used in biomonitoring of insecticides and household cleaning products used for vector control, as it presented excellent sensitivity response to Deltamethrin $>$ Alpha-Cypermethrin $>$ Vegetable oil $>$ Lambda-cyhalothrin $=$ Lambda-cyhalothrin (two formulations). Snail $P$. canaliculata can be used in biomonitoring of insecticides and adhesive spreader used for vector control, presenting evident response to the products tested.
\end{abstract}

Keywords: biomonitoring; environmental safety; public health; household cleaning.

Autor para correspondência: Karollina Pereira Leonel - Centro Universitário da Fundação Educacional de Barretos - Avenida Professor Roberto Frade Monte, 389 - CEP: 14783-226 - Barretos (SP), Brasil E-mail: karollleone1307@gmail.com

Recebido em: 28 de julho de 2016

Aceito para publicação em: 21 de novembro de 2016

http://dx.doi.org/10.4322/1980-0029.062016 


\section{Introdução}

No Brasil, o controle e as doenças vetoriais ocorrem em razão da urbanização da população, da transformação do caráter rural dessas doenças na transmissão urbana ou periurbana e da descentralização das atividades de seu controle para municípios (TAUIL, 2006).

Dentre os vetores de doenças urbanas, o controle do mosquito Aedes aegypti, transmissor do vírus da dengue, zika e chikungunya, tem sido um enorme desafio, especialmente pelas características de seu ciclo reprodutivo (DIAS et al., 2015; MANEERAT e DAUDÉ, 2016). Apenas o vírus da dengue infecta anualmente em torno de 390 milhões de pessoas em todo o mundo (BHATT et al., 2013). O único e principal elo vulnerável de sua cadeia de transmissão, com os recursos científicos e tecnológicos hoje disponíveis, é o vetor. Assim, é necessário melhorar o controle vetorial nos municípios infestados com $A$. aegypti, pois ainda não se dispõe de tratamento etiológico ou de uma vacina eficaz (TAUIL, 2006; MANEERAT e DAUDÉ, 2016).

A. aegypti é um inseto que pode ser considerado quase cosmopolita, pois o principal fator ambiental em sua distribuição é a temperatura. Os limites desse fator norte e sul em sua distribuição variam da isotérmica de janeiro $\left[1,7^{\circ} \mathrm{C}\left(35^{\circ} \mathrm{F}\right)\right]$ e julho $\left[23,9^{\circ} \mathrm{C}\left(75^{\circ} \mathrm{F}\right)\right]$ nos Estados Unidos. Na Europa, sua distribuição corresponde à isotérmica de janeiro $\left[4,5^{\circ} \mathrm{C}\left(40^{\circ} \mathrm{F}\right)\right]$ e julho $\left[23,9^{\circ} \mathrm{C}\left(75^{\circ} \mathrm{F}\right)\right]$. $\mathrm{O}$ limite do sul da América do Sul seria dado pela linha que interliga a cidade de Tacna, no sul do Peru $\left(17^{\circ} 36^{\prime} \mathrm{S}, 70^{\circ} 12^{\prime} \mathrm{W}\right)$, com a cidade de

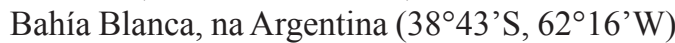
(SIMOY et al., 2015), sendo considerado um organismo de distribuição comum nas regiões subtropicais e tropicais do mundo, como a China e outras áreas da Ásia (GUO et al., 2016).

O programa de controle baseia-se no uso de produtos químicos antilarvais, inseticidas para controle de formas adultas (THONGWAT e BUNCHU, 2015) e eliminação mecânica dos criadouros, quando possível (THOMÉ et al., 2010). O principal problema da utilização contínua de inseticidas, larvicidas e ovicidas é a ocorrência de resistência, tendo sido relatada especialmente em formas adultas de $A$. aegypti, aos inseticidas organoclorados (LUMJUAN et al., 2005), organofosforados e piretroides (OCAMPO et al., 2011; THONGWAT e BUNCHU, 2015) e à deltametrina
(MARRIEL et al., 2016). Igualmente, as larvas já manifestam resistência aos produtos organofosforados, como Temephos $^{\circledR}$ (GRIGORAKI et al., 2016).

A aplicação de inseticida deveria ser reservada a situações de surtos epidêmicos, tendo em vista sua baixa eficácia, o impacto ao meio ambiente e custo elevado, porém, em razão das condições de epidemias que ocorrem em vários países, o manejo de produtos que visam ao controle do mosquito adulto tem sido uma sistemática frequente (THONGWAT e BUNCHU, 2015; FERNÁNDEZSALAS et al., 2015).

No controle de vetores, os piretroides são utilizados em mosquitos adultos. Trata-se de compostos químicos sintéticos similares às substâncias naturais piretrinas produzidas por Chrysanthemum cinerariaefolium e C. coccineum e cujo principal mecanismo de ação consiste em manter os canais de sódio abertos nas membranas neurais dos insetos, causando excitação contínua do neurônio, o que provoca a morte do inseto (SAILLENFAIT et al., 2015). Assim, os efeitos do uso de produtos no controle da dengue têm sido monitorados, especialmente em saúde pública, porém poucos são os estudos de monitoramento dos possíveis efeitos ambientais para organismos não alvos da aplicação ou os possíveis efeitos ambientais desses produtos (BRAGA e VALLE, 2007).

Contudo, a aplicação sistematizada desses produtos pode atingir os corpos hídricos superficiais carregados pelas águas pluviais urbanas, entre outros fatores. Os organismos aquáticos estão em constante contato com substâncias estranhas a seu organismo, as quais se denominam xenobióticos. Estudos ecotoxicológicos visam subsidiar e orientar práticas de monitoramento e efeito ambiental de produtos químicos, como os utilizados no controle de vetores.

A utilização de ensaios ecotoxicológicos para avaliar a contaminação ambiental é reconhecida como uma ferramenta complementar à análise química (CROUAU e MOIA, 2006), que busca conhecer a consequência da liberação de substâncias químicas no ambiente sobre os organismos nele existentes (CHASIN e PEDROZO, 2004), tendo por finalidade compreender como são nocivas ou deletérias a organismos vivos (KNIE e LOPES, 2004), com a verificação dos efeitos letais, morfológicos, comportamentais, fisiológicos, citogenéticos e 
bioquímicos nos organismos expostos aos poluentes (NEUHAUSER et al., 1985).

A utilização de bioindicadores de exposição da fauna e flora brasileira pode ser uma alternativa viável para o monitoramento de produtos domissanitários utilizados no controle de vetores. Sua utilização permite a avaliação integrada dos efeitos ecológicos causados por múltiplas fontes de poluição. Os bioindicadores mais utilizados são aqueles capazes de diferenciar fenômenos naturais de estresses de origem antrópica relacionados a fontes de poluição pontuais ou difusas (CONTI e CECCHETTI, 2001). Assim, o caramujo $P$. canaliculata, que é originário da região tropical da América do Sul (ESTEBENET e MARTÍN, 2003) e apresenta dieta composta de perifíton, macrófitas, detritos e matéria orgânica, pode ser empregado no monitoramento desses produtos. Esse bioindicador foi utilizado em estudos de toxicidade aguda de produtos à base de íon cobre (CE50;96 h = 0,07 mg.L $\left.\mathrm{L}^{-1}\right)$ e azadiractina $\left(1,68 \mathrm{mg} . \mathrm{L}^{-1}\right)$ (VENTURINI et al., 2008), do antibiótico florfenicol (CE50;48 $\mathrm{h}>100,0 \mathrm{mg} . \mathrm{L}^{-1}$ ), do inseticida toltrazuril (7,59 mg.L $\mathrm{L}^{-1}$ ) (FLORÊNCIO et al., 2014) e do herbicida Imazapyr ${ }^{\circledR}$ com CE50;48 h $=5,89$ mg. $\mathrm{L}^{-1}$ (CRUZ et al., 2015).

Esse bioindicador é um organismo comum nos principais corpos hídricos brasileiros, especialmente em ambientes lênticos, com contato direto com o sedimento desses ambientes. Esse organismo foi selecionado para avaliar produtos utilizados no controle de vetores da dengue. Assim, os objetivos deste estudo foram determinar a toxicidade aguda (CE50;48 h) de lambda-cialotrina, deltametrina, alfacipermetrina e espalhante adesivo óleo vegetal para o caramujo ( $P$. canaliculata) utilizado como bioindicador de exposição em ambiente aquático.

\section{Material e Métodos}

Os exemplares de caramujo (P. canaliculata) utilizados nos experimentos provieram do setor de cultivo do Laboratório de Ecotoxicologia e Eficácia de Agrotóxicos (LEEA), do Centro Universitário da Fundação Educacional de Barretos (Unifeb), no estado de São Paulo, onde se realizam a reprodução e a manutenção dessa espécie para ser empregada em testes de toxicidade, em caixas com capacidade para $500 \mathrm{~L}$ de água, proveniente do sistema de abastecimento urbano, onde passa por um procedimento de desclorificação por um período estacionário de dez dias, sedimento de fundo composto de latossolo, areia e matéria orgânica $\left(1 ; 1 ; 1\right.$ v.v $\left.\mathrm{v}^{-1}\right)$, fluxo contínuo de água e alimentação à base de macrófitas flutuantes.

Os produtos comerciais utilizados foram Brasão ${ }^{\circledR}$, inseticida piretroide (lambda-cialotrina a 2,5\% m/v), Veget'Oil ${ }^{\circledR}$, inseticida/espalhante adesivo (óleo vegetal a $93 \%$ v/v), Fendona ${ }^{\circledR}$ (alfacipermetrina a $6 \%$ ), Demand ${ }^{\circledR}$ (lambda-cialotrina a 2,5\% m/v) e Decis ${ }^{\circledR}($ deltametrina a $2,5 \% \mathrm{~m} / \mathrm{v})$.

\section{Aclimatação e ensaios de toxicidade aguda}

Para determinar a toxicidade aguda (CE50;48 h), os caramujos foram previamente aclimatados por um período de cinco dias. A aclimatação foi realizada em sala de bioensaio com temperatura entre $25,0 \pm 2,0^{\circ} \mathrm{C}$, iluminação de 1.000 lux e fotoperíodo de 12 horas, em aquários de dez litros, com sistema de aeração contínuo promovido por bombas de ar. Nesse período, os animais foram alimentados com a macrófitas Egeria najas, uma vez ao dia.

Os ensaios de toxicidade foram conduzidos no sistema estático, com dois litros de água, sem substituição desta, durante o período de exposição, que foi de 48 horas. A avaliação de imobilidade foi realizada em 24 e 48 horas.

Os ensaios preliminares de toxicidade aguda com lambda-cialotrina, deltametrina, alfacipermetrina e óleo vegetal foram realizados com seis concentrações e um controle, nos quais foram determinados os intervalos de concentração que causaram zero e $100 \%$ de mortalidade, tendo os intervalos obtidos sido utilizados para montar os ensaios definitivos.

Nos ensaios definitivos, determinou-se a toxicidade aguda (CE50;48 h) de lambda-cialotrina $\left(\right.$ Brasão $^{\circledR}$ e Demand $\left.{ }^{\circledR}\right)$, deltametrina, alfacipermetrina e óleo vegetal. Para tanto, os animais com peso médio de 1,5 $\pm 0,30 \mathrm{~g}$ foram expostos a cinco a seis concentrações, variando de $0,01,0,05,0,1$, $0,5,1,0,3,44,11,6,36,5,118 \mathrm{mg} . \mathrm{L}^{-1}$ e um controle (sem adição de produto teste), com três réplicas e cinco caramujos por réplica, com as unidades experimentais distribuídas aleatoriamente na sala de bioensaio.

Os dados de toxicidade aguda foram submetidos à regressão linear e a estimativa da concentração letal e efetiva $50 \%$ foi obtida pelo método de Trimmed Spearman-Karber (HAMILTON et al., 1977) e a classificação da ecotoxicidade, de acordo com a escala de Zucker e Jonhsson (1985). 


\section{Resultados e Discussão}

Para o inseticida lambda-cialotrina, nas duas formulações, não foi possível estimar a concentração efetiva de $50 \%$, sendo a CE50;48 h superior a $118,00 \mathrm{mg} . \mathrm{L}^{-1}$ para o caramujo $P$. canaliculata (Tabela 1). Assim, tais formulações foram consideradas praticamente não tóxicas conforme a classificação ecotoxicológica de Zucker e Jonhsson (1985).

A concentração efetiva de lambda-cialotrina não foi estimada pela ausência de mortalidade dos organismos testes. Os ensaios de toxicidade foram conduzidos até a concentração limite de 100,0 mg. $\mathrm{L}^{-1}$, pois a OECD (2009) determina que os ensaios agudos devem ser realizados com a concentração limite de $100,0 \mathrm{mg} . \mathrm{L}^{-1}$, pois, se não ocorrer mortalidade até essa concentração, será possível verificar com $99 \%$ de confiança que o organismo estudado não está no grupo mais sensível à substância testada.

A baixa toxicidade de lambda-cialotrina para P. canaliculata difere da descrita para o peixe-zebra (Danio rerio), com CL50;96 h de 1,94 $\mu \mathrm{g} . \mathrm{L}^{-1}$, para o camarão (Macrobrachium nipponense), com CL50;96 h de 0,04 $\mu$ g.L.-1 (WANG et al., 2007), para a tilápia (Oreochromus niloticus), com CL50;96 h de 2,901 $\mu \mathrm{g} . \mathrm{L}^{-1}$ (PINER e ÜNER, 2012), e para o microcrustáceo (Daphnia magna) com CE50;48 h, variando de 0,1 a $0,5 \mu \mathrm{g} . \mathrm{L}^{-1}$, dependendo do $\mathrm{pH}$ do meio testado (SON et al., 2015).

Para deltametrina, na formulação Decis ${ }^{\circledR}$, a toxicidade aguda (CE50;48 h) foi de $0,45 \mathrm{mg} \cdot \mathrm{L}^{-1}$, sendo seu limite superior de $0,61 \mathrm{mg} . \mathrm{L}^{-1}$ e seu limite inferior de $0,32 \mathrm{mg} \cdot \mathrm{L}^{-1}$. Esse ingrediente ativo foi classificado como muito tóxico para o caramujo (P. canaliculata), de acordo com Zucker e Jonhsson (1985). No ensaio de toxicidade aguda, ocorreu relação entre o aumento da concentração e a mortalidade dos organismos expostos. P. canaliculata foi mais tolerante à deltametrina do que Daphnia magna

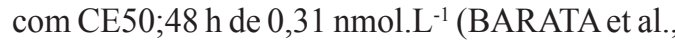
2006) e Ceriodaphnia dubia com CE50;48 h de 0,23 $\mu \mathrm{g} . \mathrm{L}^{-1}$ (SHEN et al., 2012).

Deltametrina foi mais tóxica para $P$. canaliculata do que para Vibrio fischeri com concentração efetiva (CE50;30 min) superior a 39,9 mg. $\mathrm{L}^{-1}$ (HERNANDO et al., 2007).

$\mathrm{Na}$ avaliação da alfacipermetrina na formulação Fendona ${ }^{\circledR}$ a $6 \%$, via subcutânea, a toxicidade aguda (CE50;48 h) foi de 28,76 mg. $\mathrm{L}^{-1}$, com limites superior de $45,98 \mathrm{mg} . \mathrm{L}^{-1}$ e inferior de 17,99 mg. $\mathrm{L}^{-1}$ (Tabela 1), sendo classificado como pouco tóxico para o caramujo ( $P$. canaliculata), conforme demonstraram Zucker e Jonhsson (1985). P. canaliculata foi mais tolerante a alfacipermetrina do que o peixe Poecilia reticulata com CL50;96 h de 9,43 $\mu \mathrm{g} . \mathrm{L}^{-1}$ (YILMAZ et al., 2004) e do que Ceriodaphnia dubia com CE50;48 h de $0,06 \mu \mathrm{g} . \mathrm{L}^{-1}$ (SHEN et al., 2012). A presença de 4,0, 8,0 e 12,0 $\mu \mathrm{g} . \mathrm{L}^{-1}$ de cipermetrina na água causou amento significativo na ovoposição de $149 \%, 257 \%$ e $295 \%$, em relação ao controle, do caramujo Lymnaea acuminata (TRIPATHI e SINGH, 2004), indicando que não há somente efeito agudo da presença desse piretroide na água, mas também subletal ou crônico.

$\mathrm{Na}$ avaliação do óleo vegetal, na formulação Veget'Oil ${ }^{\circledR}$ a $6 \%$, a toxicidade aguda (CE50;48 h) foi de 70,62 mg. $\mathrm{L}^{-1}$, sendo seu limite superior de 99,76 mg. $\mathrm{L}^{-1}$ e seu limite inferior de 49,99 mg.. $\mathrm{L}^{-1}$. Esse ingrediente ativo foi classificado como pouco tóxico para o caramujo ( $P$. canaliculata), conforme indicaram Zucker e Jonhsson (1985). Para o óleo vegetal, também ocorreu relação entre o aumento da concentração e a mortalidade dos organismos expostos.

O óleo vegetal foi menos tóxico para o caramujo do que surfactantes agrícolas para o

Tabela 1. Ecotoxicidade dos produtos para controle de vetores para $P$. canaliculata.

\begin{tabular}{|c|c|c|c|c|}
\hline Produtos testados & $\begin{array}{c}\text { Limite } \\
\text { superior }\end{array}$ & $\begin{array}{l}\text { CE50;48 h } \\
\left(\mathrm{mg}^{\left.-L^{-1}\right)}\right.\end{array}$ & Limite inferior & $\begin{array}{c}\text { Classificação } \\
\text { ecotoxicológica }\end{array}$ \\
\hline Lambda-cialotrina (Brasão ${ }^{\circledR}$ ) & - & $>118,0$ & - & Praticamente não tóxica \\
\hline $\begin{array}{l}\text { Lambda-cialotrina (Demand }{ }^{\circledR} \\
2,5 \mathrm{CS} \text { ) }\end{array}$ & - & $>118,0$ & - & Praticamente não tóxica \\
\hline Deltametrina $\left(\right.$ Decis $\left.^{\circledR}\right)$ & 0,61 & 0,45 & 0,32 & Muito tóxica \\
\hline $\begin{array}{l}\text { Alfacipermetrina (Fendona }{ }^{\circledR} \text { a } \\
6 \% \text {, via SC) }\end{array}$ & 45,98 & 28,76 & 17,99 & Pouco tóxica \\
\hline Óleo vegetal $\left(\right.$ Veget’Oil $^{\circledR}$ ) & 99,76 & 70,62 & 49,99 & Pouco tóxico \\
\hline
\end{tabular}

-: não estimado. 
peixe mato-grosso (Hyphessobrycon eques), com concentração letal de 50\% (CL(I)50;96 h) de 3,29 mg.L $\mathrm{L}^{-1}$ para $\mathrm{Agral}^{\circledR}$, de 8,21 mg.L $\mathrm{L}^{-1}$ para Aterbane $^{\circledR}$ BR, de 2,34 mg.L $\mathrm{L}^{-1}$ para Energic ${ }^{\circledR}$, de 4,37 mg.L $\mathrm{L}^{-1}$ para Gotafix ${ }^{\circledR}$ e de 3,38 mg.L. ${ }^{-1}$ para Fixade $^{\circledR}$ (CARRASCHI et al., 2011).

De forma geral, os inseticidas piretroides utilizados no controle de vetores e avaliados neste estudo são praticamente não tóxicos ou pouco tóxicos para $P$. canaliculata, assim como o espalhante óleo vegetal, permitindo sua utilização sem causar efeito agudo ou dano ao ambiente aquático, exceto deltametrina, que foi considerada muito tóxica para o bioindicador. Contudo, a utilização sistematizada de inseticidas tem contribuído para a ocorrência de outro problema de controle ambiental, a resistência de $A$. aegypti (VONTAS et al., 2012), sendo necessário monitoramento constante da presença dos inseticidas presentes no ambiente em concentrações subletais.

\section{Conclusão}

O caramujo $P$. canaliculata pode ser empregado no biomonitoramento de inseticidas e espalhante adesivo utilizados no controle de vetores, com resposta sensível aos produtos testados.

\section{Referências}

BARATA, C.; BAIRD, D.J.; NOGUEIRA, A.J.A.; SOARES, A.M.V.M.; RIVA, M.C. Toxicity of binary mixtures of metals and pyrethroid insecticides to Daphnia magna Straus. Implications for multisubstance risks assessment. Aquatic Toxicology, v. 78, n. 1, p. 1-14, 2006. PMid:16510198. http:// dx.doi.org/10.1016/j.aquatox.2006.01.013.

BHATT, S.; GETHING, P.W.; BRADY, O.J.; MESSINA, J.P.; FARLOW, A.W.; MOYES, C.L.; DRAKE, J.M.; BROWNSTEIN, J.S.; HOEN, A.G.; SANKOH, O.; MYERS, M.F.; GEORGE, D.B.; JAENISCH, T.; WINT, G.R.W.; SIMMONS, C.P.; SCOTT, T.W.; FARRAR, J.J.; HAY, S.I. The global distribution and burden of dengue. Nature, v. 496, n. 7446, p. 504-507, 2013. PMid:23563266. http://dx.doi.org/10.1038/nature12060.

BRAGA, I.A.; VALLE, D. Aedes aegypti: histórico do controle no Brasil. Epidemiologia e Serviços de Saúde, v. 16, n. 2, p. 113-118, 2007.

CARRASCHI, S.P.; PATRÍCIA CUBO, P.; SCHIAVETTI, B.L.; SHIOGIRI, N.S.; CRUZ,
C.; PITELLI, R.A. Efeitos tóxicos de surfactantes fitossanitários para o peixe mato grosso (Hyphessobrycon eques). Acta Scientiarum: Biological Sciences, v. 33, n. 2, p. 191-196, 2011.

CHASIN, A.A.M.; PEDROZO, M.F.M. O estudo de toxicologia. In: AZEVEDO, F.A.; CHASIN, A.A.M. (editor). As bases toxicológicas da ecotoxicologia. São Carlos: RiMa/InterTox, 2004. 340 p.

CONTI, M.E.; CECCHETTI, G. Biological monitoring lichers as bioindicators of air pollution assessment: a review. Environmental Pollution, v. 114, n. 3, p. 471-492, 2001. PMid:11584645. http://dx.doi.org/10.1016/S0269-7491(00)00224-4.

CROUAU, Y.; MOIA, C. The relative sensitivity of growth and reproduction in the springtail, Folsomia candida, exposed to xenobiotics in the laboratory: an indicator of soil toxicity. Ecotoxicology and Environmental Safety, v. 64, n. 2, p. 115-121, 2006. PMid:16040122. http://dx.doi.org/10.1016/j. ecoenv.2005.06.002.

CRUZ, C.; SILVA, A.F.; SHIOGIRI, N.S.; GARLICH, N.; PITELLI, R.A. Imazapyr herbicide efficacy on floating macrophyte control And ecotoxicology for non-target organisms. Planta Daninha, v. 33, n. 1, p. 103-108, 2015. http://dx.doi.org/10.1590/ S0100-83582015000100012.

DIAS, W.O.; WANNER, E.F.; CARDOSO, R.T. A multiobjective optimization approach for combating Aedes aegypti using chemical and biological alternated step-size control. Mathematical Biosciences, v. 269, p. 37-47, 2015. PMid:26362231. http:// dx.doi.org/10.1016/j.mbs.2015.08.019.

ESTEBENET, A.L.; MARTÍN, P.R. Shell interpopulation variation and its origin in Pomacea canaliculata (Gastropoda: Ampullaridae) from southern pampas, Argentina. Journal of Molluscan Studies, v. 69, n. 4, p. 301-310, 2003. http://dx.doi. org/10.1093/mollus/69.4.301.

FERNÁNDEZ-SALAS, I.; DANIS-LOZANO, R.; CASAS-MARTÍNEZ, M.; ULLOA, A.; BOND, J.G.; MARINA, F.C.; LOPEZ-ORDONEZ, T.; ELIZONDO-QUIROGA, A.; TORRES-MONZÓN, J.A.; DÍAZ-GONZALEZ, E.E. Historical inability to control Aedes aegypti as a main contributor of fast dispersal of chikungunya outbreaks in Latin America. Antiviral Research, v. 124, p. 30-42, 
2015. PMid:26518229. http://dx.doi.org/10.1016/j antiviral.2015.10.015.

FLORÊNCIO, T.; CARRASCHI, S.P.; CRUZ, C.; SILVA, A.F.; MARQUES, A.M.; PITELLI, R.A. Bioindicadores neotropicais de ecotoxicidade e risco ambiental de fármacos de interesse para aquicultura. Boletim do Instituto de Pesca, v. 40, n. 4, p. 569-576, 2014.

GRIGORAKI, L.; BALABANIDOU, V.; MERISTOUDIS, C.; MIRIDAKIS, A.; RANSON, H.; SWEVERS, L.; VONTAS, J. Functional and immunohistochemical characterization of CCEae3a, a carboxylesterase associated with temephos resistance in the major arbovirus vectors Aedes aegypti and Ae. albopictus. Insect Biochemistry and Molecular Biology, v. 74, p. 61-67, 2016. PMid:27180726. http://dx.doi.org/10.1016/j. ibmb.2016.05.007.

GUO, X.X.; LI, C.X.; ZHANG, Y.M.; XING, D.; DONG, Y.D.; ZHANG, H.D.; QIN, C.F.; ZHAO, T.Y. Vector competence of Aedes albopictus and Aedes aegypti (Diptera: Culicidae) for the DEN2FJ10 and DEN2-FJ11 strains of the dengue 2. virus in Fujian, China. Acta Tropica, v. 161, p. 86-90, 2016. PMid:27260668. http://dx.doi.org/10.1016/j. actatropica.2016.05.018.

HAMILTON, M.A.; RUSSO, R.C.; THURSTON, V. Trimmed Spearman-Karber method for estimating medial lethal concentrations in toxicoty bioassays. Environmental Science \& Technology, v. 7, n. 7, p. 714-719, 1977. http://dx.doi.org/10.1021/ es60130a004.

HERNANDO, M.D.; DE VETTORI, S.; MARTÍNEZ BUENO, M.J.; FERNÁNDEZ-ALBA, A.R. Toxicity evaluation with Vibrio fischeri test of organic chemicals used in aquaculture. Chemosphere, v. 68, n. 4, p. 724-730, 2007. PMid:17292447. http:// dx.doi.org/10.1016/j.chemosphere.2006.12.097.

KNIE, J.L.W.; LOPES, E.W.B. Testes toxicológicos: métodos, técnicas e aplicações. Florianópolis: FATMA/GTZ, 2004.

LUMJUAN, N.; MCCARROLL, L.; PRAPANTHADARA, L.A.; HEMINGWAY, J.; RANSON, H. RANSON, H. Elevated activity of an Epsilon class glutathione transferase confers DDT resistance in the dengue vector, Aedes aegypti.
Insect Biochemistry and Molecular Biology, v. 35, n. 8, p. 861-871, 2005. PMid:15944082. http:// dx.doi.org/10.1016/j.ibmb.2005.03.008.

MANEERAT, S.; DAUDÉ, E. A spatial agentbased simulation model of the dengue vector Aedes aegypti to explore its population dynamics in urban areas. Ecological Modelling, v. 333, p. 66-78, 2016. http://dx.doi.org/10.1016/j.ecolmodel.2016.04.012.

MARRIEL, N.B.; TOMÉ, H.V.V.; GUEDES, R.C.N.; MARTINS, G.F. Deltamethrin-mediated survival, behavior, and oenocyte morphology of insecticide-susceptible and resistant yellow fever mosquitos (Aedes aegypti). Acta Tropica, v. 158, p. 88-96, 2016. PMid:26943998. http://dx.doi. org/10.1016/j.actatropica.2016.02.021.

NEUHAUSER, E.F.; LOEHR, R.C.; MILLIGAN, D.R.; MALECKI, M.R. Toxicity of metals to the earthworns Eisenia foetida. Biology and Fertility of Soils, v. 1, n. 3, p. 149-152, 1985. http://dx.doi. org/10.1007/BF00301782.

OCAMPO, C.B.; SALAZAR-TERREROS, M.J.; MINA, N.J.; MCALLISTER, J.; BROGDON, W. Insecticide resistance status of Aedes aegypti in 10 localities in Colombia. Acta Tropica, v. 118, n. 1, p. 37-44, 2011. PMid:21300017. http://dx.doi. org/10.1016/j.actatropica.2011.01.007.

ORGANIZATION FOR ECONOMIC COOPERATION AND DEVELOPMENT - OECD. Draft guidance document: the threshold approach for acute fish toxicity testing. Paris, 2009. 4 p.

PINER, P.; ÜNER, N. Oxidative and apoptotic effects of lambda-cyhalothrin modulated by piperonyl butoxide in the liver of Oreochromis niloticus. Environmental Toxicology and Pharmacology, v. 33, n. 3, p. 414-420, 2012. PMid:22387602. http:// dx.doi.org/10.1016/j.etap.2012.01.001.

SAILLENFAIT, A.M.; NDIAYE, D.; SABATÉ, J.P. Pyrethroids: Exposure and health effects - An update. International Journal of Hygiene and Environmental Health, v. 218, n. 3, p. 281-292, 2015. PMid:25648288. http://dx.doi.org/10.1016/j. ijheh.2015.01.002.

SHEN, M.F.; KUMAR, A.; DING, S.Y.; GROCKE, S. Comparative study on the toxicity of pyrethroids, a-cypermethrin and deltamethrin to Ceriodaphnia dubia. Ecotoxicology and Environmental Safety, v. 
78, p. 9-13, 2012. PMid:22189213. http://dx.doi. org/10.1016/j.ecoenv.2011.07.018.

SIMOY, M.I.; SIMOY, M.V.; CANZIANI, G.A. The effect of temperature on the population dynamics of Aedes aegypti. Ecological Modelling, v. 314, p. 100-110, 2015. http://dx.doi.org/10.1016/j. ecolmodel.2015.07.007.

SON, J.; HOOVEN, L.A.; HARPER, B.; HARPER, S.L. Effect of $\mathrm{pH}$ and ionic strength on exposure and toxicity of encapsulated lambda-cyhalothrin to Daphnia magna. The Science of the Total Environment, v. 538, p. 683-691, 2015. PMid:26327636. http:// dx.doi.org/10.1016/j.scitotenv.2015.08.035.

TAUIL, P.L. Perspectivas de controle de doenças transmitidas por vetores no Brasil. Revista da Sociedade Brasileira de Medicina Tropical, v. 39, n. 3, p. 275-277, 2006. PMid:16906253. http:// dx.doi.org/10.1590/S0037-86822006000300010.

THOMÉ, R.C.A.; YANG, H.M.; ESTEVA, L. Optimal control of Aedes aegypti mosquitoes by the sterile insect technique and insecticide. Mathematical Biosciences, v. 223, n. 1, p. 12-23, 2010. PMid:19735668. http://dx.doi.org/10.1016/j. mbs.2009.08.009.

THONGWAT, D.; BUNCHU, N. Susceptibility to temephos, permethrin and deltamethrin of Aedes aegypti (Diptera: Culicidae) from Muang district, Phitsanulok Province, Thailand. Asian Pacific Journal of Tropical Medicine, v. 8, p. 14-18, 2015.

TRIPATHI, P.K.; SINGH, A. Toxic effects of cypermethrin and alphamethrin on reproduction and oxidative metabolism of the freshwater snail, Lymnaea acuminata. Ecotoxicology and Environmental Safety, v. 58, n. 2, p. 227-235, 2004. PMid:15157577. http://dx.doi.org/10.1016/j. ecoenv.2003.12.006.

VENTURINI, F.P.; CRUZ, C.; PITELLI, R.A. Toxicidade aguda do sulfato de cobre e do extrato aquoso de folhas secas de nim para o caramujo (Pomacea canaliculata). Acta Scientiarum: Biological Sciences, v. 30, n. 2, p. 179-184, 2008.

VONTAS, J.; KIOULOS, E.; PAVLIDI, N.; MOROU, E.; DELLA TORRE, A.; RANSON, $\mathrm{H}$. Insecticide resistance in the major dengue vectors Aedes albopictus and Aedes aegypti. Pesticide Biochemistry and Physiology, v. 104, n. 2, p. 126-131, 2012. http://dx.doi.org/10.1016/j. pestbp.2012.05.008.

WANG, W.; CAI, D.J.; SHAN, Z.J.; CHEN, W.L.; POLETIKA, N.; GAO, X.W. Comparison of the acute toxicity for gamma-cyhalothrin and lambda-cyhalothrin to zebra fish and shrimp. Regulatory Toxicology and Pharmacology, v. 47, n. 2, p. 184-188, 2007. PMid:17052827. http:// dx.doi.org/10.1016/j.yrtph.2006.09.002.

YILMAZ, M.; GÜL, A.; ERBAŞLI, K. Acute toxicity of alpha-cypermethrin to guppy (Poecilia reticulata, Pallas, 1859). Chemosphere, v. 56, n. 4, p. 381-385, 2004. PMid:15184001. http://dx.doi. org/10.1016/j.chemosphere.2004.02.034.

ZUCKER, E.; JONHSSON, S.L. Hazard evaluation division: standard evaluation procedure: acute toxicity test for freshwater fish. Washington USEPA, 1985. 17 p. (USEPA Publication, 540/9-85-006). 